Fecha de recepción: diciembre 2018 Fecha de aceptación: marzo 2019 Versión final: abril 2019

\section{El Diseño sistémico transdisciplinar para el desarrollo sostenible neguentrópico de la producción artesanal}

Miguel Angel Rubio Toledo*

Resumen: La globalización es un proceso sistémico mundial que enfatiza dos grandes directrices, las tramas de la comunicación mundial y las interacciones económicas, especialmente aquellas relacionadas con la movilidad de los recursos financieros y comerciales. Este sistema-mundo integra, como elementos de relación, a una gran cantidad de ramas y derivaciones que fungen como rizomas - procesos entrópicos vinculantes de orígenes y destinos- que abarcan y se entrelazan complejizando los fenómenos a modo de bucle. En el proceso de Diseño transdisciplinar concurren relaciones de producción simbólica y material de diversas áreas creativas. Una de ellas es la artesanía, cuya condición particular de distinguirse paradójica y peyorativamente dado el origen étnico de sus productores, le significa una baja rentabilidad económica y menoscabo cultural y, por añadidura, un abandono sistémico por parte de los propios artesanos -entropía-, fenómeno observado al menos en México. Ello permite concebir al problema artesanal como un sistema complejo, cuyos elementos interdefinidos facultan su estudio desde enfoques disciplinarios integrados en un rizoma común -transdisciplina- en dispositivos multidisciplinarios, compartiendo marcos conceptuales comunes. Es menester entonces, no sólo el análisis de la condición entrópica de la artesanía, sino la posibilidad neguentrópica para el desarrollo artesanal desde los atributos transdisciplinares del Diseño.

Palabras claves: Diseño sistémico - Transdisciplina - Desarrollo sostenible - Neguentropía - Artesanía

[Resúmenes en inglés y portugués en las páginas 61 - 75]

${ }^{(*)}$ Doctor en Ciencias Sociales, Maestro en Artes Visuales, Licenciado en Diseño Gráfico, con Especialidad en Diseño Estratégico. Profesor Investigador de Tiempo Completo del Centro de Investigación en Arquitectura y Diseño de la Universidad Autónoma del Estado de México. Perfil PRODEP y Miembro del Sistema Nacional de Investigadores. Sus Líneas Generales de Aplicación del Conocimiento se relacionan con la producción y el consumo simbólico estético, el Diseño Estratégico y la Teoría del Diseño, sobre las cuales ha escrito 5 libros y más de 50 artículos indizados y capítulos de libro. 


\section{Introducción}

En la época actual, se puede advertir una resignificación acelerada de la práctica de vida cotidiana y su implicación en la cosmovisión de los grupos sociales como causa y consecuencia de la condición rizomática sistémica de la globalización. Hay diversos autores que han enfatizado esta condición en diversas áreas del conocimiento tales como Cândida en Educación (2010), Barone sobre Políticas públicas (2001), Martínez acerca de Filosofía política (2003), Varona sobre Epistemología (2016), entre otros. Se perciben fenómenos tradicionales y nuevos, otros mundiales y locales, aun cuando todos ostentan la condición de ser recurrentes, antagónicos y complementarios, condiciones necesarias para todo sistema (Morin, 2001), para este caso el sociocultural.

Estos fenómenos implican la necesidad de observarlos como ramificaciones vinculantes de un sistema de fenómenos (principio del rizoma) de menor o mayor extensión o amplitud (Deleuze, 2002), que permiten su estudio desde diversas disciplinas y ciencias de manera aislada. En términos generales, lo anterior se percibe en la perpetua y creciente migración de las zonas rurales a las ciudades (Muñoz, 2002). También se advierten en la oscilación de las expresiones culturales producto de la modificación de los subsistemas de creencias (Mercado, 2008). Del mismo modo, se observan en la falta de compromisos reales de desarrollo por parte de los gobiernos (Posada, 2018).

Asimismo, estas situaciones se perciben en las desfavorables aperturas comerciales de mercado (Ocegueda, 2007), sin olvidar el desarrollo masivo de las hipertecnologías con sus consecuencias en las patologías sociales tales como la nomofobia, la depresión, la adicción, la cibercondria (Caro, 2017), entre muchos otros. Estos fenómenos, junto con otras condiciones sociales y culturales (mismos que no se detallan en este documento en virtud de que no es el objeto del mismo) han dado lugar a la pauperización económica de las comunidades culturales tradicionales, tal como ilustra Novelo sobre los artesanos en México

La situación de pobreza de la gran mayoría de los artesanos mexicanos indígenas, que comparten con otras mayorías rurales y urbanas del país, se debe a un sinfín de causas estructurales, políticas y de falta de realismo en los planes, más asistencialistas que de desarrollo, que han puesto en marcha los distintos gobiernos del país en un marco de economía capitalista subdesarrollada y de política poco democrática (Novelo, 2002, p. 173).

Resulta fundamental conocer y reflexionar sobre el modo en que se construyen y deconstruyen socialmente las diversas formas de cultura actuales, la forma científica de abordarlas y proponer en consecuencia posibilidades desde el Diseño. Se pretende poner a consideración diversos argumentos desde el diseño definido como sistema transdisciplinar para la posibilidad de paliar los impactos negativos en las prácticas sociales y permitir el desarrollo sostenible de lo cultural artesanal de modo neguentrópico.

Los fundamentos sistémicos muestran que los elementos, las estructuras mundiales y sus interrelaciones son principios claves para comprender los cambios que ocurren a nivel social, político, de división de la producción y de particulares condiciones nacionales y regionales (Smith y White, 1992). La globalización así entendida, puede ser observada 
como una Teoría del desarrollo donde un mayor nivel de integración tiene lugar entre las diferentes regiones del mundo, resultado del uso de las actuales tecnologías mediáticas, cuyo nivel de integración afecta las condiciones sociales de los países.

Una de las implicaciones necesarias y particulares de la globalización es su énfasis en los elementos de comunicación y aspectos culturales. Esta condición distingue la interacción de individuos y grupos de manera masiva, enfatizando el intercambio de contenidos, recreando las posibilidades de realidad en distintas geografías, a modo de aldea global desde lo local. Desde una perspectiva socio cultural, los nuevos productos para la comunicación están desarrollando un patrón de intercambio e interconexión mundiales, es decir, de su práctica social. Así, se vuelve necesario señalar que esta singularidad resulta fundamental para el desarrollo de países con enorme arraigo cultural como México.

El capital cultural (Bourdieu, 2008) deviene en un valor único e ilimitado que permite que las comunidades subalternas que generan cultura tradicional popular (como la artesanía hecha generalmente, en México por indígenas), puedan generar subsistemas de circuitos para sus productos, reduciendo las prácticas de venta que suelen resultar endebles en virtud de los costos y las ganancias económicos. Así, el valor del trabajo (producto y proceso) se incrementa al ser distinguido como experiencia histórica simbólica con fines de desarrollo económico y, por añadidura social (o neguentropía sistémica), como lo han señalado ya diversos autores (Pedraza, 2012; Lazcano, 2005; García, 1989; Castro, 2002). Es a partir de la posibilidad de otorgar el necesario valor a las prácticas, usos y costumbres histórico-simbólicos de los sujetos que crean los objetos artesanales que el pensamiento estratégico permite diagnosticar problemas conceptuados como sistemas complejos (orden, desorden, interacción y organización) desde el Diseño como transdisciplina o eje epistémico con la finalidad estratégica de coadyuvar a paliar las condiciones desfavorables de las comunidades indígenas artesanales en sus contextos. Este diseño sistémico transdisciplinar sugiere -entre sus diversas posibilidades- la revisión necesaria, vital y trascendental de los potenciales procesos de desarrollo en comunidades vulnerables principalmente, aunque no únicamente.

De tal suerte, el proceso estratégico de diseño sistémico procura la traducción del ya existente alto valor simbólico-cultural transformándolo en un alto valor económico -valor de uso y valor de cambio hacia el valor de signo simbólico (Baudrillard, 2010)- en una primera instancia. En una segunda, la reificación de los objetos a través de su valor de uso simbólico sugiere que se perpetúen las manifestaciones de cosmovisión tradicionales tales como los ritos, los mitos, las expresiones en las prendas de vestir, accesorios, y maquillajes, entre muchas otras (Montemayor, 2000), con el objeto de fortalecer a las comunidades en su imaginario colectivo de autoestima social.

\section{Globalización y consumo cultural}

En los sistemas sociales, es menester distinguir las condiciones históricas y su desarrollo para observar los fenómenos vinculados con la producción social de sentido y material. Resulta necesario mencionar que la postura social sistémica aquí retomada requiere la comprensión de que el conocimiento de los sistemas sociales depende del análisis de las 
condiciones sociales no sólo por su cultura material, sino principalmente por su conocimiento y las relaciones derivadas. Esto es, no se revisan únicamente los objetos, hechos y sus interpretaciones (Latour, 2008), sino la manera en que éstos influyen o modifican los modos de producción del conocimiento a través de la comunicación para dar lugar -a manera de bucle- a otro orden u organización susceptible de observar -autorreferencia(Luhmann, 2006).

Arizpe (2002) señala que a partir de los años setenta del siglo XX, en los países occidentales se empieza a discutir la noción de cultura y el papel que desempeña en múltiples aspectos del desarrollo humano, cuyos contenidos en Latinoamérica, a diferencia de los modelos europeos, se han tratado de insertar en los procesos de integración regional, aun cuando se consideraba como factor y no como fundamento de interacción colectiva de comunidad. En los años noventa del mismo se precisó trascender lo económico pero sin abandonar su importancia. En 1989 se amplía la idea de desarrollo humano con criterios que miden la libertad social, económica y política, las oportunidades individuales para la salud, la educación, la productividad, el disfrutar del respeto personal, los derechos humanos, entre otros (Programa de Naciones Unidas para el Desarrollo, 2019).

La importancia que le otorga el Programa de Naciones Unidas para el Desarrollo (PNUD) a los procesos de bienestar y desarrollo, los concibe no como estados acabados de los sujetos, sino como su construcción permanente orientada a fines. Es decir, se considera la interrelación simbiótica de los sujetos como semiosis inacabada de la interacción entre las posibilidades de los valores en la realidad. Lo anterior se puntualizó en el fortalecimiento de un nuevo paradigma de desarrollo que fuera más allá del crecimiento del producto interno bruto, se asignó a la Comisión Independiente de Naciones Unidas sobre Cultura y Desarrollo realizar un informe. Nuestra Diversidad Creativa fue el nombre que recibió el primer informe en el que se sostenía, entre otras cosas, que: "El respeto por las culturas se tiene que entender entonces dentro de un marco mayor que comprende los derechos humanos, la democracia, la equidad de género y la equidad entre generaciones" (Arizpe, 2002, p. 101). Por su parte, Hopenhayn (2002), sostiene que los conceptos como Estado-Nación, territorio e identidad nacional se ven trastocados en virtud de que la globalización económica y cultural borra las fronteras y las identidades asociadas con ellas, del mismo modo que las acentúa sistémicamente (recurrente, antagónico y complementario). Aún en la segunda mitad del siglo XX era común normalizar los signos simbólicos como estrategia del poder hegemónico desde el Estado, sin embargo, la mediatización tecnológica actual ha permitido gradualmente ir paliando estándares que hoy se discuten como posibilidades de zonas dinámicas de libertades y respetos distintos.

Lo anterior se traduce sistémicamente a partir del conflicto en el orden moderno, donde se genera el caos o desorden a través de las mediaciones o interacciones informacionales que darán lugar a su vez a otra organización u orden, y que servirá de partida para el siguiente bucle de realidad, para este caso del poder del Estado, las hipertecnologías y la cultura

La asimetría entre emisores y receptores en el intercambio simbólico se convierte en un problema político, de lucha por ocupar espacios de emisión/ recepción por constituirse en interlocutor visible y en voz audible. Mientras 
avanza, a escala global, un status quo que racionaliza económicamente por el lado del capitalismo, y políticamente por el lado de las democracias formales, adquiere mayor conflictividad el ámbito de la cultura y la identidad. (Hopenhayn, 2002, p. 63)

Así, aumenta la visibilidad política del campo de la afirmación cultural y de los derechos de la diferencia, pasando de las lógicas de representación a las lógicas de redes, es decir, las demandas dependen menos del sistema político que las procesa y más de los actos comunicativos que fluyen por las redes o sistemas de información actuales (Lozares, 1998; Reynoso, 2008). Se trata de poner al ciudadano en el centro de las políticas culturales, esto es, a los actores de las organizaciones civiles a través de redes. No obstante, es importante señalar, que el consumo cultural del tipo artesanal, suele ser soslayado por los contenidos hipertecnificados debido a la inercia de las industrias culturales más accesibles en estos medios de comunicación.

Las transformaciones actuales provienen de las mutaciones que atraviesa el entramado tecnológico de la comunicación, las cuales afectan la percepción que las comunidades culturales tienen de sí mismas y de sus condiciones de existencia, de sus modos de construir y dar cuenta de sus identidades de manera sistémica (opuesta, recurrente y complementaria). Esto es, la conformación de mega corporaciones globales virtuales concentran los vehículos de los contenidos a través de la opinión pública y la apertura a nuevos espacios de constitución ciudadana e identitaria, a través del intercambio como forma de apertura sistémica (Barbero, en Mata, 1999).

México, dadas las particulares condiciones contextuales históricas, es una región fuertemente rica en culturas y tradiciones, tanto prehispánicas como mestizas, donde una de las expresiones sincréticas más conocidas y valoradas de dichas culturas es la artesanía (Sales, 2013; Novelo, 2002; Arizpe, 2011). Estos objetos se han desarrollado de manera vasta por cientos de años desde la época prehispánica como mezcla cultural hispánica e indígena en dos vertientes. Por un lado, como objeto utilitario para realizar actividades cotidianas como vasijas, canastas, juguetes, entre muchos otros; y por otro, como objeto suntuario, simbólico o religioso, en virtud de las profundas creencias de los diversos pueblos.

En México las artesanías son producidas principalmente por los grupos étnicos existentes $^{1}$, lo que le otorga a estos objetos la característica de ser realizados de acuerdo con saberes históricos, transferidos en la mayoría de las ocasiones de manera más práctica que verbal, de generación a generación desde épocas ancestrales. No obstante, es necesario señalar que a través del tiempo se han realizado modificaciones a los objetos con base en las condiciones socioeconómicas y contextuales de las propias comunidades ${ }^{2}$. Además, existe un elevado (indeterminado) número de artesanías dadas las características de sus materiales, procesos y usos. Sus diferencias de producción material y simbólica implican características regionales culturales y sociales, dado que los materiales son generalmente endémicos y sus connotaciones simbólicas obedecen a las propias cosmovisiones étnicas. El gobierno mexicano creó en 1974 el Fondo Nacional para el Fomento a las Artesanías (FONART), el cual actualmente realiza ferias, exposiciones, concursos, talleres y sirve como agencia de empleo para los artesanos. No obstante, a pesar de ser una instancia con planes y 
objetivos definidos, el apoyo no resulta suficiente para la promoción, premios y distribución, en tanto una gran parte de los recursos económicos de programas sociales, la mayor parte de éstos se quedan en los funcionarios públicos que son parte de los canales de distribución encargados de entregarlos a las comunidades (Transparencia Internacional, 2017). Por su parte, a finales del siglo XX el Banco Nacional de Comercio Exterior (BANCOMEXT) desarrolló estrategias para apoyar a las comunidades artesanales, particularmente para la promoción y distribución sus productos en el extranjero. Sin embargo, dadas las características socioculturales étnicas de los artesanos (Sales, 2013; Lazcano, 2005), sólo un pequeño grupo de éstos ha tenido la oportunidad de utilizar las ventajas para exportar. A pesar de dichos esfuerzos, el sistema de las artesanías en México se observa como un arte popular menor (Freitag, 2014), es decir, su concepción histórica y elaboración son estrictamente valorados por la destreza de la técnica empleada.

De acuerdo con el FONART, cerca de 13 millones de personas en México han realizado alguna actividad relacionada con la producción artesanal de un aproximado de $125 \mathrm{mi}$ llones de mexicanos, es decir, alrededor del $10 \%$ de la población mexicana ha realizado artesanías en algunas de sus múltiples formas, procesos o materiales, lo que representa un porcentaje considerable y sistémicamente una actividad bastante dinámica. El consumo de las artesanías también ostenta una condición peculiar, donde el mercado interno es mucho mayor que el del consumidor exterior o mercado de exportación, a pesar de los esfuerzos institucionales para esta actividad, lo que quiere decir que el habitante mexicano consume muchos más objetos artesanales que los extranjeros (Secretaría de Desarrollo Social, 2017).

\section{El diseño sistémico transdisciplinar}

El diseño sistémico transdisciplinar surge como una ampliación epistémica del diseño estratégico en su práctica y teoría. Particularmente como una optimización integral de las aplicaciones de la versión del diseño estratégico aquí vertida. Algunos de los antecedentes pueden observarse en la postura de diseño centrado en el usuario o human-centered design, así como en otras formas conocidas del diseño universal divulgado por Victor Papanek (2014). Del mismo modo, en el libro Pensamiento de diseño (Design thinking) de Peter G. Rowe publicado en 1987 (Rowe, 1991), se enfatiza el uso sistemático de procedimientos de solución de problemas, utilizado principalmente por arquitectos y planeadores urbanos, constituye quizás el antecedente más directo del diseño estratégico.

En términos generales, los sistemas complejos, implican la constitución de un umbral organizador del conocimiento que vincula una descripción del proceso de investigación, así como la descripción de la descripción (Luhmann, 1996) enfatizando la articulación y la integración entre ambos, además de la distinción y lo antagónico. Se busca la desregulación de la información observada, la dislocación semántica en lo simbólico como nuevo orden. La organización es ese nuevo orden, es la explicación contingente que se distingue en un orden y un desorden vinculando los elementos y las estructuras del sistema como interacciones, ostentando un significado de evolución y revolución. 
La perspectiva de realidad germina del propio observador, implicando el escrutinio teorizado de un "trazo de la realidad que incluye aspectos físicos, biológicos, sociales, económicos y políticos" (García, 2006, p. 47). Se persigue la circunscripción de los elementos de condición interdefinible con los límites no definidos, así como con las interrelaciones internas y externas del sistema. Los contornos entre los niveles (subsistemas, suprasistemas, eco-sistemas, macrosistemas) no son evidentes en cuanto a su orden o flujo de información y pueden ser intercambiables entre ellos. En lo particular son intercambiables a partir del modo de organización social o cultural y la propia necesidad del observador para distinguir la realidad sistémica establecida.

Para la investigación del diseño sistémico, se trata principalmente de "la revisión de la jerarquización (horizontal) de diversos sistemas y sus posibles niveles dentro del aparato de estudio del grupo a observar, mediante el análisis de las interrelaciones y las articulaciones entre dichos sistemas o niveles" (Rubio, 2018, p. 75). Esto significa que los procesos de semiosis entre los factores, elementos o subsistemas son estudiados en lo intangible donde las expresiones formales son las posibilidades de revelación de los procesos complejos de interacción. La importancia para la investigación del diseño se observa en la revisión de las interacciones entre los componentes del sistema cultural, más que en los elementos del constituyentes.

La investigación del diseño con apoyo de la descripción densa (Geertz, 2001), observa la necesidad de indagar las interacciones, los órdenes, los desórdenes y las organizaciones de los objetos, contextos físicos, y el ruido, la información, la redundancia y la organización de los estados psíquicos de la comunidad observada.

Entendida como sistemas en interacción de signos interpretables (que, ignorando las acepciones provinciales, yo llamaría símbolos), la cultura no es una entidad, algo a lo que puedan atribuirse de manera causal acontecimientos sociales, modos de conducta, instituciones o procesos sociales; la cultura es un contexto dentro del cual pueden describirse todos esos fenómenos de manera inteligible, es decir, densa (Geertz, 2001, p. 27).

Se crea un bucle rizomático de conocimiento u organización sistémica al tiempo que se indaga, a partir de la interacción de los fenómenos como una espiral abierto que se clausura operativamente (Luhmann, 2006) produciendo saber contingente. Dicho bucle resultante produce organización que genera la realidad desde las interrelaciones entre el sujeto y el objeto. Se trata de la contingencia de los hechos en un particular momento lógicamente teorizados como expresión de dicha experiencia de descripción densa. Ello sirve de fundamento para la génesis del concepto complejo que dará como resultado el diseño del sistema de objetos orientado a fines. Las posibilidades de interacciones simbólicas resultantes entre los objetos y los sujetos servirán a su vez como base de investigación para el diseño bajo el mismo espectro pero diacrónicamente.

En este proceso complejo, dadas las características del propio objeto de investigación para el diseño, se deconstruyen permanentemente aparatos teóricos y empíricos de matices disciplinarios de diversa índole, dirigidos ex profeso para una meta epistemología específica. 
El discurso transdisciplinario derivado como bucle, involucra la necesidad ontológica del diseño para proyectar sistemas de vida "en la medida en que tiene en cuenta no sólo las cosas sino también los seres y su relación con otros seres y con las cosas" (Nicolescu, 2009, p. 88). Se torna estratégico para el sistema mundo como un sistema complejo, advertir toda la posible información presente en cierta situación o evento, no obstante, existe siempre la posibilidad de la incertidumbre prevista de las respuestas temporales.

El diseño sistémico opera como un rizoma (Deluze y Guattari, 2002), esto es, como transdisciplina, cuyas vinculaciones tangenciales científicas se trenzan a manera de bucle alrededor del proyecto de diseño como eje. Las aproximaciones teóricas y empíricas científicas, tecnológicas y artísticas se tratan como herramentales de razonamiento y proposición del proyecto de diseño. Se constituyen sistémicamente en la investigación, en la conceptualización, en la propuesta, en la materialización y en el seguimiento y control de los proyectos de diseño.

Este proceso de diseño sistémico se distingue como un aparente problema finiquitado de algún fenómeno y hasta un aleatorio final que en realidad es un bucle rizomático que da pie al desarrollo de procesos recursivos, antagónicos y complementarios no finitos. Se fortalece la investigación como descripción densa, se observa al diseño como transdisciplina o eje rector del proyecto desde el diseño, y se pretende que las soluciones sean nuevas organizaciones sistémicas y subsistémicas -bucles neguentrópicos- que den lugar a posibles nuevos eventos.

Resulta necesario señalar que, la diferencia entre el diseño estratégico tradicional y el diseño sistémico transdisciplinar aquí propuesto, se observa en los determinantes complejos de interrelaciones de la investigación, de la conceptualización, de la proyección, de la solución y del control de los procesos de diseño. Esta perspectiva requiere del enfoque de los procesos de comunicación entre las distintas formas de relación de los sujetos y los objetos (semiosis), allende la proxémica y le otorgue a dicha interactividad un carácter fundamental sincrónico simbólico no permanente. Ello permite la construcción de sistemas de experiencias de vida significativas (emocionales, intelectuales, entre otras), desde la caracterización representativa de las particularidades de sus sistemas simbólicos como la interactividad de los metasistemas de ritos, mitos, tabúes, etc. (Rubio, 2018).

El diseño estratégico nace en el seno de la globalización de las tecnologías de comunicación que permean los subsistemas de objetos que produce el diseño. Pero no se agota ahí, sino que pretende influir en todo el sistema de la realidad, para efectos de integrar cada una de las posibilidades de esta disciplina al ámbito del desarrollo de la red de valor de las comunidades y organizaciones, cuyo concepto principal se observa en el sistemaproducto. El diseño estratégico sistémico, al que se refiere este trabajo, implica procesos complejos no lineales de manera general de cada subsistema fundado teóricamente en estrategias para el desarrollo adecuado de los sujetos en sus comunidades.

Se trata de la deconstrucción abductiva ${ }^{3}$ del proceso de diseño en vinculación con los posibles resultados en diversos escenarios. Ello permite involucrar desde la filosofía de la organización en donde se toman decisiones, la proyección de los bienes, la creación conceptual y física o virtual del resultado. Esto significa que se trata no sólo de productos aislados, sino de un cuerpo integrador de productos, servicios y comunicaciones, desde una visión estratégica, en otras palabras, un sistema de subsistemas. 
La complejidad de lo estratégico estriba y está en relación directa a la cada vez mayor posibilidad de escenarios alternativos posibles sobre los que actuar. Actualmente en todos ellos son múltiples los actores que interactúan, de fisonomías y características cambiantes. Por lo tanto, la dificultad de dominar lo estratégico estriba en la capacidad de garantizar que se están valorando los "mejores resultados posibles" en las diversas situaciones posibles. (Fernández, 2010, p. 33)

Por su parte, Viladàs (como se citó en Fernández, 2010, p. 11) señala que la evolución del diseño hacia otros ámbitos de acción se ha dado en virtud de la capacidad de conducirse en "escenarios complejos, la habilidad para leer indicios y anticipar tendencias, la facilidad para visualizar conceptos y para comunicarlos de manera eficiente, y todo ello centrado en el usuario y adaptándose a la frontera de posibilidades de cada proyecto". Así, el diseño responde sistemáticamente a problemas en diversos ámbitos tales como la educación, la economía, la cultura, la filosofía, la política, entre muchas otras.

Sostiene Fernández (2010) que lo que le otorga el sentido estratégico al diseño, "es la necesidad de definir los objetivos del proyecto a ser alcanzados a largo, mediano y corto plazo de acuerdo a un orden de prioridades, de metas a cumplir dentro de determinados márgenes de riesgo" (p. 20). El diseño estratégico debe partir de un concepto complejo que describa de manera puntual las propiedades materiales e inmateriales del producto, su grado de innovación y, especialmente, el sentido que pretende transmitir. Este concepto u organización que el autor denomina identidad genética, es construido a partir de un sistema de redes de conexiones -interacciones sistémicas para Morin (2001)- en la cual se conectan todas las acciones y acontecimientos que participan del proyecto, actuando como selector de prioridades y caminos proyectuales, al tiempo de crear identidad al interior de la organismo.

\section{Desarrollo sostenible neguentrópico de la condición sistémica artesanal}

Se propone entonces, argumentar los proyectos de diseño como resultado del análisis y síntesis de una investigación en diseño profunda y extensa. Desde el diseño, se encuentran elementos y criterios subsistémicos rizomáticos de otras áreas del conocimiento lo que le otorga el carácter de transdisciplina. Ello permite realizar una descripción profunda del consumidor (desorden sistémico) con objeto de conocer (orden sistémico) los elementos que lo identifican simbólicamente (interacción sistémica) y construir conceptualmente al objeto de diseño (organización sistémica).

Así, no sólo es importante entender la estructura social desde la producción cultural o científica, sino realizar un análisis de la interacción. En dicho análisis subyacen condiciones para legitimar las posiciones en términos de una transferencia de consumo, según una fracción de los intelectuales orgánicos gramscianos (Bourdieu, 2008). Es decir, estudiar las representaciones o expresiones culturales pero también la forma de producción material (interacciones de los discursos humanos y objetuales), los modos de reproducción cultu- 
ral y las formas en que se organiza socialmente y su construcción de sentidos (reorganización conceptual). En estos contenidos se encuentra la producción simbólica.

Se procede desde lo simbólico en tanto la cultura no se trata únicamente de las prácticas, sino la abstracción de sus interacciones, en virtud de que son pautas que permiten actuar y que al ponerse en práctica se convierten en expresiones. Éstas, ostentan un sentido, puesto que las prácticas y los valores son interpretados por los involucrados. Estas exégesis, construidas social e históricamente, da lugar a que diversos grupos encuentren diferentes sentidos de una determinada práctica y valor. Lo antedicho a su vez, le otorga a la cultura el sentido de desorden sistémico. "Las únicas formas de diferenciación sistémica viables se encuentran las regionales, explicadas por las diferencias de participación y de reacción ante las estructuras dominantes del sistema de la sociedad mundial" (Luhmann, 2006, p. 126).

Las economías emergentes -como México- se encuentran en proceso de mediatización y en la búsqueda de crecimiento económico. Aún cuando en el marco de estas economías, lo importante sea la posibilidad de analizar no sólo las ventajas de los intercambios de productos económicos y culturales, sino del conocimiento de los procesos de producción colectiva de significados no estandarizada (semiosis diferenciada). Es decir, los procesos de producción colectiva particularizada en cuanto a sus sentidos tal como lo es la artesanía. Esto es justamente lo que enfatiza la riqueza simbólica para convertirla en económica como estrategia neguentrópica desde el diseño transdisciplinar. Este eje de análisis permite la resignificación de los componentes, dispositivos y procesos en los sistemas de artesanías, principalmente en términos cognitivos y metacognitivos como principio organizador.

Lo anterior, otorga fuerza tanto a la articulación como a la integración de la realidad y sistema mundo de vida de los objetos (artesanías) y los sujetos (artesanos). Es aquí donde se observa lo sistémico complejo, en la revisión de los procesos de vinculación de los componentes y los sistemas de los mundos de vida en integración. La organización sistémica de la realidad sesga el paradigma hacia su propio beneficio, esto es, el valor simbólico como valor principal jerárquicamente por encima del económico. Se plantea el tipo ideal y se complementa con teorías de escenarios para observar la pertinencia neguentrópica de la complejidad del sistema cultural en sus diversas presentaciones de la realidad desde los subsistemas y a partir de sus características de desorden, orden, caos e incertidumbre. La cultura entonces, conserva su carácter fundamental para la identidad de un país y su contribución esencial a la cohesión social. No obstante, como efecto de la globalización, es percibida cada vez más nítidamente como sinónimo de la imagen identitaria de un país en el escenario global. La imparable combinación de las expresiones de la cultura con el desarrollo de las tecnologías y de las telecomunicaciones, se convierte no ya en un contenido con valor añadido, sino en el elemento clave para el desarrollo sostenible. Los procesos de globalización facultan al diseño como una disciplina necesaria para el tratamiento de las comunidades y organizaciones públicas y privadas.

El diseño transdisciplinar así observado, permite diagnosticar problemas observados como sistemas complejos (orden, desorden, interacción y organización) a partir de la organización de interconexiones como semiosis. Así, desde el capital cultural se enfatiza el valor simbólico del sistema de artesanías, permitiendo que las comunidades subalternas que generan cultura tradicional generen autopoiéticamente subsistemas de circuitos para 
sus productos. Los fundamentos del diseño estratégico transdisciplinar en la teoría de producción simbólica puede constituirse por las estructuras dinámicas sistémicas de cohesión conceptual que determinan su validez pragmática a partir de la mediación de la acción social (Habermas, 1999).

La imbricación de los medios autopoiéticos estratégicos para la conservación y desarrollo de patrones sociales, debe ser ahora precisada en función de la propia práctica social y económica sostenible. Si bien los elementos de creación de valor sugieren ahora que los componentes se dirijan hacia el dispositivo fundamental de la economía, es decir, el valor, también es cierto que estos valores deben ser fundados en dispositivos simbólicos como antecedente de contenido dirigidos a partir del diseño transdisciplinar. En esta clave cobra sentido el diseño para una economía política del signo simbólico autopoiético intersubjetivo orientado a fines, esto es, hacia el beneficio social.

\section{Listas de Referencias Bibliográficas}

Alcántara S. (16 de septiembre de 2018). Panistas piden prohibir venta de imitaciones de artesanías. Diario El Universal. Recuperado de https://www.eluniversal.com.mx/nacion/ politica/panistas-piden-prohibir-venta-de imitaciones-de-artesanias

Alcazar G., A., y Marissa R. (Abril 13 de 2018). Las artesanías de Chiapas se ven amenazadas por imitaciones, la mayoría hechas en China. Global Press Journal. Recuperado de https:// globalpressjournal.com/americas/mexico/chiapas-artisans-face-major-threat-knockoffhandicrafts-mostly-made-china/es/

Amador T., J. (2018, 22 de septiembre). Defensa del arte popular contra la piratería. Revista Proceso. Número 2185. Recuperado de https://www.proceso.com.mx/552053/defensadel-arte-popular-contra-la-pirateria

Arizpe S. L. (2002). "Cultura o voluntad política: cómo construir el pluralismo en México”. En Béjar R., y Rosales H. (Coord.), La identidad nacional mexicana como problema político y cultural. Los desafíos de la pluralidad (pp. 95-118). Cuernavaca, México: Centro de Investigaciones Multidisciplinarias, UNAM.

Arizpe S.L. (2011). Cultura e identidad. Mexicanos en la era global. Revista de la Universidad de México. Nueva época. (92). Recuperado de http://www.revistadelauniversidad.unam. $\mathrm{mx} / 9211 /$ arizpe/92arizpe.html

Barone, M. (2001). "Globalización y Posmodernidad: Encrucijada para las políticas sociales del nuevo milenio". En Reunión de Expertos sobre Globalización, Cambio Tecnológico y Equidad de Género. Comisión Económica para América Latina y el Caribe - CEPAL, Fondo de Desarrollo de las Naciones Unidas para la Mujer - UNIFEM, Sao Paulo, Brasil. Recuperado de https://www.cepal.org/mujer/noticias/noticias/0/8260/posmodernidad.pdf

Baudrillard, J. (2010). Crítica de la economía política del signo. Madrid, España: Siglo XXI Editores.

Bourdieu, P. (2008). Capital cultural, escuela y espacio social. Ciudad de México: Siglo XXI Editores. 
Caro M., M. M (2017). Adicciones tecnológicas: ¿Enfermedad o conducta adaptativa?. Revista de Ciencias Médicas Cienfuegos Medisur. 15 (2), 251-260. Recuperado de http:// scielo.sld.cu/scielo.php?script=sci_arttext\&pid=S1727-897X2017000200014.

Cândida M., M. (2010). Transdisciplinariedad y educación. Rizoma freireano. Instituto Paulo Freire de España. 6 (6). Recuperado de http://www.rizomafreireano.org/transdisciplinaridade-e-educacao--maria-candida-moraes

Castro S., J. (2002). Reivindicación estética del arte popular. Revista de Filosofía. Universidad Complutense de Madrid. 27 (2). 431-451.

Deleuze, G. y Guattari, F. (2002). Mil Mesetas. Capitalismo y esquizofrenia. Valencia, España: Pre-textos.

Fernández G.J. (2010). Diseño estratégico. Guía metodológica. Madrid, España: Fundación PRODINTEC y FEDER.

Freitag, V. (2014). Entre arte y artesanía: elementos para pensar el oficio artesanal en la actualidad. El Artista. (11), 129-143. Recuperado de https://www.redalyc.org/ $\mathrm{html} / 874 / 87432695007$

García C., N. (1989). Las culturas populares en el capitalismo. Ciudad de México: Nueva Imagen.

García, R. (2006). Sistemas complejos. Conceptos, método y fundamentación epistemológica de la investigación interdisciplinaria. Barcelona, España: Gedisa.

Geertz, C. (2001). La interpretación de las culturas. Barcelona, España: Gedisa.

Habermas, J. (1999). La teoría de la acción comunicativa, volúmenes 1 y 2. Madrid, España: Taurus.

Hopenyayn, M. (2002). El reto de las identidades y la multiculturalidad. Revista de cultura: Pensar Iberoamérica. Recuperado de http://www.oei.es/pensariberoamerica/

Instituto Nacional de los Pueblos Indígenas (2019). Informes del Instituto Nacional de los Pueblos Indígenas. Recuperado de https://www.gob.mx/inpi/documentos/informes-delinstituto-nacional-de-los-pueblos-indigenas?idiom=es

Latour B. (2008). Reensamblar lo social: una introducción a la teoría del Actor-Red. Buenos Aires, Argentina: Manantial.

Lazcano A. N. J. (2005). El trabajo artesanal. Una estrategia de reproducción de los mazahuas en la Ciudad de México. México: Instituto Nacional de las Mujeres.

Lozares, C. (1996). La teoría de redes sociales. Papers. Revista de Sociología. (48). 103-126. Recuperado de https://papers.uab.cat/article/view/v48-lozares

Luhmann, N. (1996). Introducción a la teoría de Sistemas. Ciudad de México: Antrophos, ITESO, UIA.

Luhmann, N. (2006). La sociedad de la sociedad. (Trad. Javier Torres Nafarrete). Ciudad de México: Herder-UIA.

Martínez de B., A. (2003). Tópicos para una filosofía política: globalización, poder, identidad y cuestión colonial en América Latina. Utopía y Praxis Latinoamericana. Revista Internacional de Filosofía Iberoamericana y Teoría Social. 8 (20), 67-79.

Mata, María Cristina (1999). De la cultura masiva a la cultura mediática. Diálogos de la comunicación, (56). 80-92 
Mercado M., J. (2008). Las consecuencias culturales de la migración y cambio identitario en una comunidad tzotzil, Zinacantán, Chiapas, México. Agricultura, sociedad y desarrollo, 5 (1), 19-38. Recuperado de http://www.scielo.org.mx/scielo.php?script=sci_arttext\&pi $\mathrm{d}=$ S187054722008000100002\&lng=es\&tlng=es.

Montemayor, C. (2000). La cosmovisión de los pueblos indígenas actuales. Desacatos. Revista de Ciencias Sociales, (5), 95-106. Recuperado de https://www.redalyc.org/ $\mathrm{html} / 139 / 13900507$

Morin, E.(2001). El Método I. La naturaleza de la Naturaleza. Madrid, España: Cátedra.

Muñoz J. A. C. (2002). Efectos de la globalización en las migraciones internacionales. Papeles de población, 8 (33), 9-45. Recuperado de http://www.scielo.org.mx/scielo. php?script=sci_arttext\&pid=S140574252002000300002\&lng=es\&tlng=es

Novelo O. V. (2002). Ser indio, artista y artesano en México. Espiral. IX (25). Recuperado de https://www.redalyc.org/articulo.oa?id=13802506

Nicolescu, B. (2009). La transdisciplinariedad-manifiesto. Ciudad de México: Multiversidad Mundo Real.

Ocegueda H., J. M. (2007). Apertura comercial y crecimiento económico en las regiones de México. Investigación económica, 66 (262), 89-137. Recuperado de http://www.scielo. org.mx/scielo.php?script=sci_arttext\&pid=S018516672007000400089\&lng=es\&tlng=es

Papanek, V. (2014). Diseñar para el mundo real. Ecología humana y cambio social. Madrid, España: POL.LEN.

Pedraza, C., L. A. (2012). La tradición del maque en Uruapan, Michoacán. Historia de una tecnología artesanal. Ciudad de México: Centro de Estudios de las tradiciones de El Colegio de Michoacán.

Posada, M. (27 de febrero de 2018). Razón de la pobreza: fracaso en las políticas públicas dicen especialistas. Diario La Jornada,. Recuperado de https://www.jornada.com.mx/ ultimas/2018/02/27/razon-de-la-pobreza-fracaso-en-las-politicas-publicas-dicenespecialistas-2581.html

Programa de Naciones Unidas para el Desarrollo (2019). Objetivos del desarrollo humano. Recuperado de http://www.mx.undp.org/content/mexico/es/home/ourwork/povertyreduction/in_depth/desarrollo-humano.html

Reynoso, C. (2008). Hacia la complejidad por la vía de las redes: Nuevas lecciones epistemológicas. Desacatos, (28), 17-40. Recuperado de http://www.scielo.org.mx/scielo. php?script=sci_arttext\&pid=S1607-050X2008000300004\&lng=es\&tlng=es.

Rowe, P. (1991). Design thinking. Massachusetts, EUA: MIT.

Rubio T. M. A. (2018). Consideraciones para la investigación simbólica en desde los sistemas simbólicos". Cuadernos del Centro de Estudios en Diseño y Comunicación, 21 (82), 69-85. Recuperado de https://fido.palermo.edu/servicios_dyc/publicacionesdc/vista/ detalle_publicacion.php?id_libro=722.

Sales, H., F. J. (2013). Las artesanías en México. Situación actual y retos. (Comp). Ciudad de México: Centro de Estudios Sociales y de Opinión Pública. LXII Legislatura de la Cámara de Diputados. http://biblioteca.diputados.gob.mx/janium/bv/cesop/lxii/ art_mex_sitact_re.pdf 
Secretaría de Desarrollo Social (2017). Las artesanías en la Encuesta Nacional de Consumo Cultural de México. 2017. Recuperado de https://www.gob.mx/fonart/documentos/lasartesanias-en-la-encuesta-nacional-de-consumo-cultural-de-mexico-enccum

Smith, D. \& White, D. (1992). Structure and dynamics of the global economy. Oxford, EUA: Social Forces.

Transparencia Internacional (2017). Índice de percepción de la corrupción. 21 de febrero de 2018. Recuperado de https://www.transparency.org/news/feature/corruption_perceptions_index_2017

Varona D., F. (2012). El pensamiento complejo y la transdisciplinariedad. Fenómenos emergentes de una nueva racionalidad. Revista Facultad de Ciencias Económicas, XX (1). Recuperado de http://www.scielo.org.co/pdf/rfce/v20n1/v20n1a16.pdf

\begin{abstract}
Globalization is a systemic world process, that emphasize two major guidelines, global communication systems and economic interactions, especially those related to the mobility of financial and commercial resources. In this world-system, a large number of branches and derivations that act as rhizomes -entropic linking processes of origins and destinies- that span and intertwine systemically, complicate the phenomena as a loop. In the transdisciplinary design process the relations of symbolic and material production of diverse creative areas are concurred. One of these is the craftsmanship, whose particular condition of being distinguished paradoxically and pejoratively because the ethnic origin of the producers, means low profitability in economic and cultural impairment and, in addition, a systemic abandonment by the artisans themselves -entropy-, a phenomenon observed at least in Mexico. This gives the opportunity to conceive of the craft problem as a complex system, whose interdefinite elements allow the study from disciplinary approaches integrated in a common rhizome -transdiscipline- in multidisciplinary devices, sharing common conceptual frameworks. It is necessary then, not only the analysis of the entropic condition of the craftsmanship, but the neguentropic possibility for the artisanal development from the transdisciplinary attributes of the Design.
\end{abstract}

Key words: Systemic design - Transdiscipline - Sustainable development - Neguenthropy - Crafts

Resumo: A globalização é um processo sistêmico global que enfatiza duas diretrizes principais, os quadros de comunicação global e interações econômicas, especialmente aquelas relacionadas com a mobilidade dos recursos financeiros e comerciais. Neste sistema mundial são integrados como elementos da relação de um monte de galhos e ramos que servem como rizomas - processos entrópicos vinculativos de origens e destinos- estão entrelaçados cobertura e sistemicamente fenômenos complicando como um loop. No processo de Design transdisciplinar existem relações de produção simbólica e material de diversas áreas criativas. Um deles é o artesanato, cuja condição específica deve ser distinguida de forma paradoxal e pejorativa pela origem étnica de seus produtores, ele significa baixa rentabilidade econômica e prejuízo cultural e, além disso, um abandono sistêmico por artesãos 
-entropia-, um fenômeno observado, pelo menos no México. Isso dá a oportunidade de conceber o problema como um sistema cujos elementos interdefinidos permitem o estudo de abordagens disciplinares integrados em um rizoma comum -transdisciplina- em dispositivos multidisciplinares, a partilha de marcos conceituais comuns. É necessário, então, não só a análise da condição entrópica do artesanato, mas a possibilidade neguentrópica para o desenvolvimento artesanal a partir dos atributos transdisciplinares do Design

Palavras chave: Projeto Sistêmico - Transdisciplina - Desenvolvimento Sustentável Neguentropia - Artesanato

[Las traducciones de los abstracts fueron supervisadas por el autor de cada artículo] 